\title{
OPTIMAL STATE OBSERVATION USING QUADRATIC BOUNDEDNESS: APPLICATION TO UAV DISTURBANCE ESTIMATION
}

\author{
Julián CAYERO ${ }^{a, *}$, DAMiAno ROTONDO ${ }^{a, b}$, BERnARdo MORCEGO $^{a}$, ViCENÇ PUIG $^{a, b}$ \\ ${ }^{a}$ Specific Research Center CS2AC \\ Polytechnic University of Catalonia (UPC), Rbla. Sant Nebridi 22, 08222 Terrassa, Spain \\ e-mail: \{julen.cayero, damiano.rotondo, bernardo.morcego, vicenc.puig @upc.edu
}

${ }^{b}$ Institute of Robotics and Industrial Informatics (CSIC-UPC)

Polytechnic University of Catalonia (UPC), Llorens i Artigas 4-6, 08028 Barcelona, Spain

\begin{abstract}
This paper presents the design of a state observer which guarantees quadratic boundedness of the estimation error. By using quadratic Lyapunov stability analysis, the convergence rate and the ultimate (steady-state) error bounding ellipsoid are identified as the parameters that define the behaviour of the estimation. Then, it is shown that these objectives can be merged in a scalarised objective function with one design parameter, making the design problem convex. In the second part of the article, a UAV model is presented which can be made linear by considering a particular state and frame of reference. The UAV model is extended to incorporate a disturbance model of variable size. The joint model matches the structure required to derive an observer, following the lines of the proposed design approach. An observer for disturbances acting on the UAV is derived and the analysis of the performances with respect to the design parameters is presented. The effectiveness and main characteristics of the proposed approach are shown using simulation results.
\end{abstract}

Keywords: disturbance estimation, unmanned aerial vehicles (UAVs), optimal estimation and filtering, system modelling.

\section{Introduction}

The problem of state observation is very relevant in control, since in many control systems the state variables are not accessible for measurement due to either physical or economical limitations. For this reason, several works in the literature have focused on the design of state estimators (see, e.g., Zeitz, 1987; Grip et al., 2012; Zhang et al., 2012b; Chadli and Karimi, 2013; Rotondo et al., 2016; Hassani et al., 2017).

Among the approaches that have received attention from the control community in recent years, there are the ones based on quadratic boundedness (QB). Roughly speaking, QB refers to guaranteeing the boundedness of all the state trajectories of a system by means of a quadratic Lyapunov function. This concept was introduced first for nonlinear systems by Brockman and Corless (1995), and later extended to nominally linear systems (Brockman and Corless, 1998). Since then, several results have exploited this concept for the purpose

*Corresponding author of state estimation. For instance, Alessandri et al. (2004) used QB to deal with stability and design of receding-horizon estimators. Also Alessandri et al. (2006) applied QB to the design of state estimators for discrete-time linear systems with polytopic uncertainties, and that work was extended further by Zhang et al. (2012a), who considered state disturbances and measurement noise independently.

Notably, QB has been invoked to solve problems related to output feedback stabilization (Ding, 2009; 2013; Ding and Pan, 2016; Ping et al., 2017; Ping, 2017), fault tolerant control (Witczak et al., 2016) and fault estimation (Buciakowski et al., 2017b; 2017a; Witczak et al., 2017). Recently, further extensions of the QB concept have been investigated; see, e.g., the extended nonquadratic boundedness introduced by $\mathrm{Zou}$ and $\mathrm{Li}$ (2011).

A related topic which has been studied extensively for a number of years is the design of disturbance observers. The idea that, when a disturbance estimation is available, a control action can be taken to compensate 
for the influence of the disturbance has given birth to disturbance-observer-based control (DOBC), which was reviewed extensively by Chen et al. (2016). Since the 1960s, several techniques have been proposed to obtain the disturbance estimation, such as unknown input observers (Johnson, 1971), perturbation observers (Kwon and Chung, 2003), equivalent input disturbance-based estimators (She et al., 2008), extended state observers (Li et al., 2012), disturbance observers (Chen et al., 2000) and fuzzy Takagi-Sugeno filters (Chibani et al., 2017).

Dealing with disturbances and unmodelled dynamics is a crucial and critical issue in the control of unmanned aerial vehicles (UAVs). In some environments, controlling UAVs is a very challenging task, since any controller that does not take into account the disturbances may lead to undesired behaviour ranging from performance degradation to instability. For this reason, there has been some interest in applying DOBC to UAVs. For example, Ruggiero et al. $(2014 ; 2015)$ applied disturbance estimation techniques to UAVs by using a non-linear model with the generalized momentum as the state. In these works, algebraic equations involving the disturbance and unknown derivatives of the state are derived and the disturbance estimation is obtained by feeding the integral of the algebraic equations to a second order filter. On the other hand, Yüksel et al. (2014) derived a non-linear disturbance observer based on the non-linear equations of motion that use velocities and accelerations as states. The stability of the observer is demonstrated in the case of constant external disturbances (null derivative) and in the absence of noise. Note that the effectiveness of the above works is shown either by using indoor sensors, which provide very precise measurements of position and velocity, or by considering noise-free simulation scenarios. At the same time, they share the hypothesis that the disturbance is slow varying, which may misjudge real disturbances acting on the UAV.

The main contribution of this paper is to design quadratically bounded observers taking into account the existence of a trade-off between the convergence rate and the ultimate (steady-state) error bounding ellipsoid. In order to handle both objectives, a scalarized objective function with one design parameter is built, such that the design problem is made convex. The obtained design procedure ensures an optimal trade-off between transient and asymptotic behaviours, in the same spirit as in the work of Alessandri et al. (2006), but differing from it in two important aspects. First, instead of minimizing the ultimate upper bound of the error, the volume of the ellipsoid to which the error belongs is minimized, which leads to minimal error solutions. Second, while the whole state estimation is guaranteed to converge to the real state, the proposed design method allows the user to select a part of the state whose associated estimation error is minimized, which gives tighter solutions for the particular part of the state. These facts make the proposed approach different from other techniques developed for observer design, such as the ones based on $\mathcal{H}_{\infty}$ optimization (Wang et al., 1999; Abbaszadeh and Marquez, 2009) or the input-to-state stability (ISS) concept (Alessandri, 2004; 2013), which do not minimize explicitly the volume of the steady state ellipsoid's error.

The proposed approach is applied to the problem of estimating the disturbances in UAVs and simulation results are used to show its effectiveness and main characteristics. In particular, it is shown that the UAV model can be expressed in a linear form through an appropriate choice of the state variables (the linear and spin momenta) and the frame of reference (the world one). Then, the UAV model is extended by incorporating a disturbance high order model, as proposed by Kim et al. (2010), Godbole et al. (2013) and $\mathrm{Su}$ et al. (2015). The overall model is a linear system that matches the structure required by the proposed quadratically bounded observer. The model, by construction, admits measurements obtainable from on-board sensors after an appropriate filtering process and does not restrict the disturbance dynamics. However, it allows the inclusion of information about the nature of disturbances in desired derivative order. It is worth highlighting that, due to the specific model of the disturbance, the designed observer embeds a proportional multiple-integral (PMI) action, which shows the applicability of the design approach to structures such as the ones considered by, e.g., Busawon and Kabore (2001) or Koenig (2005).

This article is structured as follows. First, in Section 1 the notation used along the paper is presented. Next, Section 2 shows the derivation of the optimal observer design procedure. In Section 3, the model of the UAV is derived. Section 4 shows the results of applying the derived observer to the UAV model. Finally, the conclusions are presented in Section 5.

\section{Notation.}
$\mathbf{A}, \mathbf{B}, \ldots$
matrices
$\mathbf{I}_{n}$
$\mathbf{0}_{m \times n}$
$\mathbf{A}^{\top}$
$\mathbf{A}^{-1}$
A $>0$
$\mathbf{A} \geq 0$
$\mathbf{A}<0$
$\mathbf{A} \leq 0$
$\langle\mathbf{A}\rangle$
identity matrix of size $n \times n$
zero matrix of size $m \times n$
transpose of matrix $\mathbf{A}$
inverse of matrix $\mathbf{A}$
positive definite matrix
positive semi-definite matrix
negative definite matrix
negative semi-definite matrix
linear vector space spanned by the rows
of $\mathbf{A}$
$\boldsymbol{a}, \boldsymbol{b}, \ldots \quad$ vectors
$\|\boldsymbol{a}\| \quad$ norm of the vector $\boldsymbol{a}$
$[\boldsymbol{a}]_{\times}$
$\stackrel{\circ}{q}$ skew-symmetric matrix of vector $\boldsymbol{a}$ unit quaternion 


$\begin{array}{ll}\mathbf{R}(\stackrel{\circ}{q}) & \begin{array}{l}\text { rotation matrix given by the quaternion } \stackrel{\circ}{q} \\ \epsilon_{\mathbf{M}}(\boldsymbol{x})\end{array} \\ \begin{array}{ll}\text { ellipsoid represented by } \boldsymbol{x}^{\top} \mathbf{M} \boldsymbol{x} \leq 1 \\ \text { with } \mathbf{M}>0 \text { and symmetric }\end{array} \\ (J)^{\star} & \begin{array}{l}\text { optimal value of the scalar function } J \\ (J)^{\dagger}\end{array} \\ & \text { nadir value of the scalar function } J\end{array}$

\section{Optimal quadratic boundedness observer}

Consider the dynamic system defined as 1

$$
\left\{\begin{array}{l}
\dot{\boldsymbol{x}}=\mathbf{A} \boldsymbol{x}+\mathbf{B} \boldsymbol{u}+\mathbf{W}_{1} \boldsymbol{\eta}_{1} \\
\boldsymbol{y}=\mathbf{C} \boldsymbol{x}+\mathbf{D} \boldsymbol{u}+\mathbf{W}_{2} \boldsymbol{\eta}_{2} \\
\boldsymbol{z}=\mathbf{E}_{1} \boldsymbol{x}
\end{array}\right.
$$

where $\boldsymbol{x}=\boldsymbol{x}(t) \in \mathbb{R}^{n}$ represents the state, $\boldsymbol{u}=$ $\boldsymbol{u}(t) \in \mathbb{R}^{m}$ represents the input, $\boldsymbol{y}=\boldsymbol{y}(t) \in \mathbb{R}^{p}$ represents the output, $\boldsymbol{\eta}_{1}=\boldsymbol{\eta}_{1}(t) \in \mathbb{R}^{l_{1}}$ represents the disturbances affecting the dynamic model, $\boldsymbol{\eta}_{2}=\boldsymbol{\eta}_{2}(t) \in$ $\mathbb{R}^{l_{2}}$ represents the measurement noise and $\boldsymbol{z}=\boldsymbol{z}(t) \in \mathbb{R}^{q}$ represents a subset of the state vector. Also, the matrices $\mathbf{A} \in \mathbb{R}^{n \times n}, \mathbf{B} \in \mathbb{R}^{n \times m}, \mathbf{W}_{1} \in \mathbb{R}^{n \times l_{1}}, \mathbf{C} \in \mathbb{R}^{p \times n}$, $\mathbf{D} \in \mathbb{R}^{p \times m}, \mathbf{W}_{2} \in \mathbb{R}^{p \times l_{2}}$ and $\mathbf{E}_{1} \in \mathbb{R}^{q \times n}$ are constant with $\mathbf{E}_{1}$ representing a part of a permutation matrix.

Let the vector containing the disturbance and noise terms, denoted as $\overline{\boldsymbol{\eta}}=\left(\boldsymbol{\eta}_{1}^{\top}, \boldsymbol{\eta}_{2}^{\top}\right)^{\top}$, be bounded by the ellipsoid $\epsilon_{\mathbf{Q}}(\overline{\boldsymbol{\eta}})$, i.e.,

$$
\overline{\boldsymbol{\eta}}^{\top} \mathbf{Q} \bar{\eta} \leq 1
$$

and let the estimation of the state $\boldsymbol{x}$ be represented by $\hat{\boldsymbol{x}}$ and the error associated to the state estimation denoted as $\boldsymbol{e}=\boldsymbol{x}-\hat{\boldsymbol{x}}$. In addition, let the error of estimation of $\boldsymbol{z}$ be defined as $\boldsymbol{e}_{z}=\mathbf{E}_{1} \boldsymbol{e}$.

The main goal of this section is to design a linear observer for the state, which provides some guarantees on the signal $\boldsymbol{e}_{z}$. To this end, consider the usual linear state estimation dynamics given by

$$
\dot{\hat{\boldsymbol{x}}}=(\mathbf{A}+\mathbf{L C}) \hat{\boldsymbol{x}}+(\mathbf{B}+\mathbf{L D}) \boldsymbol{u}-\mathbf{L} \boldsymbol{y} .
$$

Then, the dynamics of the estimation error are described by

$$
\dot{\boldsymbol{e}}=(\mathbf{A}+\mathbf{L C}) \boldsymbol{e}+\mathbf{W}_{1} \boldsymbol{\eta}_{1}+\mathbf{L} \mathbf{W}_{2} \boldsymbol{\eta}_{2} .
$$

Definition 1. The system in Eqn. (4), under the conditions in Eqn. (2), is quadratically bounded for a given symmetric matrix $\mathbf{P}>0$ if the derivative of the quadratic Lyapunov function $V=\boldsymbol{e}^{\top} \mathbf{P} e$ given by

$$
\dot{V}=2 e^{\top} \mathbf{P} \dot{e}
$$

is guaranteed to be negative for values of $V>1$, i.e.,

$$
V=\boldsymbol{e}^{\top} \mathbf{P} \boldsymbol{e}>1 \Rightarrow \dot{V}=2 \boldsymbol{e}^{\top} \mathbf{P} \dot{\boldsymbol{e}}<0, \quad \forall \overline{\boldsymbol{\eta}} \in \epsilon_{\mathbf{Q}} .
$$

\footnotetext{
${ }^{1}$ Throughout the paper, the dependence of signals on time is omitted to simplify the notation.
}

The quadratic boundedness conditions stated above can be formulated as a feasibility problem.

Proposition 1. The dynamic system in Eqn. (4) with Eqn. (2) is quadratically bounded with a Lyapunov matrix $\mathbf{P}$ if there exist a symmetric matrix $\mathbf{P}>0$, a matrix $\mathbf{L}$ and a scalar $\alpha>0$ for which

$$
\left[\begin{array}{c:c:}
\mathbf{\Psi}+\alpha \mathbf{P} & \mathbf{P} \mathbf{W}_{1} \\
\hdashline \mathbf{Y W}_{2} \\
\hdashline \mathbf{W}_{1}^{\top} \mathbf{P} & -\alpha \mathbf{Q}
\end{array}\right] \leq 0
$$

with

$$
\mathbf{\Psi}=\mathbf{A}^{\top} \mathbf{P}+\mathbf{P A}+\mathbf{Y C}+\mathbf{C}^{\top} \mathbf{Y}^{\top}
$$

and

$$
\mathbf{Y}=\mathbf{P L}
$$

Proof. The time rate of change in the quadratic Lyapunov function $V(e)=e^{\top} \mathbf{P} e$ along the solutions of Eqn. (4) is given by

$$
\begin{aligned}
\dot{V} & \left(\boldsymbol{e}, \boldsymbol{\eta}_{1}, \boldsymbol{\eta}_{2}\right) \\
= & \boldsymbol{e}^{\top} \mathbf{P} \dot{\boldsymbol{e}}+\dot{\boldsymbol{e}}^{\top} \mathbf{P} \boldsymbol{e} \\
= & \boldsymbol{e}^{\top} \mathbf{P}\left((\mathbf{A}+\mathbf{L} \mathbf{C}) \boldsymbol{e}+\mathbf{W}_{1} \boldsymbol{\eta}_{1}+\mathbf{L} \mathbf{W}_{2} \boldsymbol{\eta}_{2}\right) \\
& +\left(\boldsymbol{e}^{\top}(\mathbf{A}+\mathbf{L C})^{\top}+\boldsymbol{\eta}_{1}^{\top} \mathbf{W}_{1}^{\top}+\boldsymbol{\eta}_{2}^{\top} \mathbf{W}_{2}^{\top} \mathbf{L}^{\top}\right) \mathbf{P} \boldsymbol{e} \\
= & \boldsymbol{e}^{\top}\left(\mathbf{A}^{\top} \mathbf{P}+\mathbf{P A}+\mathbf{P L C}+\mathbf{C}^{\top} \mathbf{L}^{\top} \mathbf{P}\right) \boldsymbol{e} \\
& +\boldsymbol{\eta}_{1}^{\top} \mathbf{W}_{1}^{\top} \mathbf{P} \boldsymbol{e}+\boldsymbol{e}^{\top} \mathbf{P} \mathbf{W}_{1} \boldsymbol{\eta}_{1}+\boldsymbol{\eta}_{2}^{\top} \mathbf{W}_{2}^{\top} \mathbf{L}^{\top} \mathbf{P} \boldsymbol{e} \\
& +\boldsymbol{e}^{\top} \mathbf{P} \mathbf{L} \mathbf{W}_{2} \boldsymbol{\eta}_{2} .
\end{aligned}
$$

Given the unknown nature of $\boldsymbol{\eta}_{1}$ and $\boldsymbol{\eta}_{2}$, it is not guaranteed that $\dot{V}$ is negative definite. However, note that the condition in Eqn. (2) bounds the values of $\boldsymbol{\eta}_{1}$ and $\boldsymbol{\eta}_{2}$, making $\dot{V}<0$ for sufficient large values of $\boldsymbol{e}$ and any choice of $\mathbf{L}$ that makes $\mathbf{A}+\mathbf{L} \mathbf{C}<0$.

Defining $\overline{\boldsymbol{x}}=\left(e^{\top}, \overline{\boldsymbol{\eta}}\right)^{\top}$ and making the change of variables $\mathbf{Y}=\mathbf{P L}$, the last equation can be rewritten in a quadratic form as

$$
\dot{V}(\overline{\boldsymbol{x}})=\overline{\boldsymbol{x}}^{\top}\left[\begin{array}{ccc}
\mathbf{\Psi} & \mathbf{P} \mathbf{W}_{1} & \mathbf{Y} \mathbf{W}_{2} \\
\mathbf{W}_{1}^{\top} \mathbf{P} & \mathbf{0} & \mathbf{0} \\
\mathbf{W}_{2}^{\top} \mathbf{Y}^{\top} & \mathbf{0} & \mathbf{0}
\end{array}\right] \overline{\boldsymbol{x}},
$$

which is negative semi-definite in the case when

$$
\left[\begin{array}{ccc}
\boldsymbol{\Psi} & \mathbf{P W}_{1} & \mathbf{Y} \mathbf{W}_{2} \\
\mathbf{W}_{1}^{\top} \mathbf{P} & \mathbf{0} & \mathbf{0} \\
\mathbf{W}_{2}^{\top} \mathbf{Y}^{\top} & \mathbf{0} & \mathbf{0}
\end{array}\right] \leq 0 .
$$

On the other hand, using the condition $V=$ $\boldsymbol{e}^{\top} \mathbf{P} \boldsymbol{e}>1$ and Eqn. (2), the inequality

$$
e^{\top} \mathbf{P} e>\overline{\boldsymbol{\eta}}^{\top} \mathbf{Q} \overline{\boldsymbol{\eta}}
$$


can be established or, equivalently,

$$
\overline{\boldsymbol{x}}^{\top}\left[\begin{array}{cc}
-\mathbf{P} & \mathbf{0} \\
\mathbf{0} & \mathbf{Q}
\end{array}\right] \overline{\boldsymbol{x}}<0,
$$

which implies that the symmetric matrix

$$
\left[\begin{array}{cc}
-\mathbf{P} & \mathbf{0} \\
\mathbf{0} & \mathbf{Q}
\end{array}\right]<0
$$

By applying the $S$-procedure over the inequalities in Eqns. 12 and 15, Eqn. (7) is obtained.

Proposition 2. The quadratic boundedness in Eqn. (7) implies exponential convergence of the error $e$ to the interior of $\epsilon_{\mathbf{P}}(\boldsymbol{e})$ with the decay rate driven by $\alpha$ provided that $\bar{\eta}$ fulfils Eqn. (2).

Proof. Pre and post-multiplying Eqn. (7) by $\overline{\boldsymbol{x}}^{\top}$ and $\overline{\boldsymbol{x}}$, respectively, leads to

$$
\dot{V}(\boldsymbol{e})+\alpha V(\boldsymbol{e})-\alpha \overline{\boldsymbol{\eta}}^{\top} \mathbf{Q} \overline{\boldsymbol{\eta}} \leq 0 .
$$

By using Eqn. (2), it is clear that

$$
\dot{V}(\boldsymbol{e}) \leq \alpha \overline{\boldsymbol{\eta}}^{\top} \mathbf{Q} \overline{\boldsymbol{\eta}}-\alpha V(\boldsymbol{e}) \leq \alpha(1-V(\boldsymbol{e})),
$$

which makes $\dot{V}(\boldsymbol{e})<0$ for $\boldsymbol{e}^{\top} \mathbf{P} \boldsymbol{e}>1$.

By integrating the case scenario in which $\dot{V}^{\prime}(e)=$ $\alpha\left(1-V^{\prime}(e)\right)$, it can be seen that

$$
V(\boldsymbol{e}) \leq 1+\left(V_{0}-1\right) \exp (-\alpha t),
$$

with $V_{0}=V\left(t_{0}\right)=\boldsymbol{e}\left(t_{0}\right)^{\top} \mathbf{P} \boldsymbol{e}\left(t_{0}\right)$ representing the unknown value of $V(t)$ at the initial time instant $t_{0}$. For all $t>t_{0}$, the solutions of $V(\boldsymbol{e}(t))$ are ultimately bounded by $V=1$, the errors $\boldsymbol{e}$ are guaranteed to converge to $\epsilon_{\mathbf{P}}(\boldsymbol{e})$ whenever $V_{0}>1$ and will remain inside $\epsilon_{\mathbf{P}}(\boldsymbol{e})$ as soon as they reach it or as long as $V_{0}<1$.

Moreover, the value $\alpha$ modulates the decay rate: the higher $\alpha$, the higher the convergence rate.

As a consequence of the convergence guarantee of $\boldsymbol{e}$ to $\epsilon_{\mathbf{P}}(\boldsymbol{e})$, the estimation error $\boldsymbol{e}_{z}$ will converge to the interior of the ellipsoid $\epsilon_{\mathbf{P}_{z}}\left(\boldsymbol{e}_{z}\right)$, where $\epsilon_{\mathbf{P}_{z}}\left(\boldsymbol{e}_{z}\right)$ is the resulting ellipsoid after projecting $\epsilon_{\mathbf{P}}(\boldsymbol{e})$ over the vector space generated by the rows of $\mathbf{E}_{1},\left\langle\mathbf{E}_{1}\right\rangle$ and

$$
\mathbf{P}_{z}=\mathbf{E}_{1} \mathbf{P} \mathbf{E}_{1}^{\top}-\mathbf{E}_{1} \mathbf{P} \mathbf{E}_{2}^{\top}\left(\mathbf{E}_{2} \mathbf{P} \mathbf{E}_{2}^{\top}\right)^{-1} \mathbf{E}_{2} \mathbf{P} \mathbf{E}_{1}^{\top},
$$

with $\mathbf{E}_{2}$ representing any orthogonal complement of $\mathbf{E}_{1}$, i.e., $\mathbf{E}_{1} \mathbf{E}_{2}^{\top}=\mathbf{0}$. An exhaustive derivation is provided in Appendix.

2.1. State observer design. From the statements presented above, given a fixed measure of the ellipsoid containing the terms $\bar{\eta}$, it can be established that the estimation error $\boldsymbol{e}_{z}$ will depend on
- the size of $\epsilon_{\mathbf{P}_{\mathbf{z}}}\left(\boldsymbol{e}_{z}\right)$, which represents a measure of the ultimate error bounds and can be measured through the volume of the ellipsoid (Ros et al., 2002)

$$
\operatorname{Vol}\left(\epsilon_{\mathbf{P}_{z}}\right)=\frac{B_{q}}{\sqrt{\operatorname{det}\left(\mathbf{P}_{z}\right)}}
$$

where $B_{q}$ represents the volume of the unit-ball of dimension $q$ (the dimension of the row space of $\mathbf{E}_{1}$ );

- the convergence rate represented by $\alpha$, which defines the behaviour of the transient response.

Ideally, the chosen observer gain $\mathbf{L}$ must try to maximize $\alpha$ while minimizing $\operatorname{Vol}\left(\epsilon_{\mathbf{P}_{z}}\right)$. However, both objectives are not independent but are subject to the satisfaction of the constraint in Eqn. (7). Indeed, there does not necessarily exist a solution of the problem which optimizes $\alpha$ and $\operatorname{Vol}\left(\epsilon_{\mathbf{P}_{z}}\right)$ at the same time.

Following the spirit of Alessandri et al. (2006), but re-scaling the objectives as suggested by Miettinen (1999), a parametrized mixed objective function that merges both the objectives can be defined as

$$
J_{\mu}\left(\alpha, \mathbf{P}_{z}\right)=\mu J_{1}+(1-\mu) J_{2},
$$

where $\mu$ has been introduced as a design parameter that defines the trade-off between $J_{1}$ and $J_{2}$, defined as

$$
J_{1}=\frac{\operatorname{Vol}\left(\epsilon_{\mathbf{P}_{z}}\right)-\operatorname{Vol}\left(\epsilon_{\mathbf{P}_{z}}\right)^{\star}}{\operatorname{Vol}\left(\epsilon_{\mathbf{P}_{z}}\right)^{\dagger}-\operatorname{Vol}\left(\epsilon_{\mathbf{P}_{z}}\right)^{\star}}
$$

and

$$
J_{2}=\frac{\alpha-\alpha^{\star}}{\alpha^{\dagger}-\alpha^{\star}} .
$$

The introduction of $J_{i}, i=1,2$ scales the values of the individual objectives. $J_{i} \in[0,1]$. It takes the value of 0 when the objective is completely achieved, i.e., $\alpha=\alpha^{\star}$, $\operatorname{Vol}\left(\epsilon_{\mathbf{P}_{z}}\right)=\operatorname{Vol}\left(\epsilon_{\mathbf{P}_{z}}\right)^{\star}$ and the value of 1 in the opposite case, i.e, for the nadir (worst optimal) value $\operatorname{Vol}\left(\epsilon_{\mathbf{P}_{z}}\right)=$ $\operatorname{Vol}\left(\epsilon_{\mathbf{P}_{z}}\right)^{\dagger}$ given at $\alpha=\alpha^{\dagger}$.

The optimal quadratically bounded state observer which minimizes Eqn. (21) can be designed by solving the optimization problem

$$
\min _{\alpha>0, \mathbf{P}>0, \mathbf{Y}} J_{\mu}\left(\alpha, \mathbf{P}_{z}\right),
$$

subject to Eqn. (7), for a fixed value of $\mu$. In general, the solution of Eqn. (24) is not straightforward. However, an alternative optimization problem, which shares the same solution, can be achieved in two steps:

Step 1. The constraint in Eqn. (7) is not linear due to the product of unknown variables $\alpha$ and $\mathbf{P}$. Nevertheless, it can be interpreted as an LMI for constant values of $\alpha$. Thus, an equivalent problem to Eqn. (24) can be obtained by splitting it in two nested optimization problems, one 
efficiently solvable by SDP solvers inside, a second one with a single optimization variable

$$
\min _{\alpha>0} J_{\mu}\left(\alpha, \operatorname{Vol}\left(\epsilon_{\mathbf{P}_{z}}^{+}\right)\right),
$$

with $\epsilon_{\mathbf{P}_{z}}^{+}$representing the ellipsoid whose associated quadratic matrix $\mathbf{P}_{z}^{+}$is obtained by using Eqn. (19) with $\mathbf{P}=\mathbf{P}^{+}$, which solves the optimization problem for fixed values of $\alpha$ and $\mu$ :

$$
\left(\mathbf{P}^{+}, \mathbf{Y}^{+}\right)=\arg \min _{\mathbf{P}>0, \mathbf{Y}} J_{\mu}\left(\alpha, \operatorname{Vol}\left(\epsilon_{\mathbf{P}_{Z}}\right)\right)
$$

subject to Eqn. (7).

Step 2. For fixed values of $\alpha$ and $\mu, J_{2}$ is a constant and $\mu B_{q}$ acts as a scaling parameter. Given that the square root function present in Eqn. (20) is monotonically increasing, solving Eqn. (26) is equivalent to maximizing the alternative objective function $J^{\prime}\left(\mathbf{P}_{z}\right)=\operatorname{det}\left(\mathbf{P}_{z}\right)$ subject to Eqn. (7).

Even though the determinant of $\mathbf{P}_{z}$ can be rendered convex since $\mathbf{P}_{z}$ is positive definite by construction, while its modeling from the original unknown $\mathbf{P}$ cannot, due to the product on the right-hand side of Eqn. (19). This drawback can be overcome by considering a new matrix $\mathbf{X} \geq 0$ and the constraint

$$
\mathbf{X} \leq \mathbf{P}_{z}
$$

Equation (27) can be expressed as an LMI on $\mathbf{P}$ by considering it as the Schur complement of $\mathbf{P}_{22}^{\prime}$ in $\mathbf{P}^{\prime}$, with

$$
\begin{aligned}
\mathbf{P}^{\prime} & =\left[\begin{array}{ll}
\mathbf{P}_{11}^{\prime} & \mathbf{P}_{12}^{\prime} \\
\mathbf{P}_{21}^{\prime} & \mathbf{P}_{22}^{\prime}
\end{array}\right] \\
& =\left[\begin{array}{cc}
\mathbf{E}_{1} \mathbf{P} \mathbf{E}_{1}^{\top}-\mathbf{X} & \mathbf{E}_{1} \mathbf{P} \mathbf{E}_{2}^{\top} \\
\mathbf{E}_{2} \mathbf{P} \mathbf{E}_{1}^{\top} & \mathbf{E}_{2} \mathbf{P} \mathbf{E}_{2}^{\top}
\end{array}\right]>0 .
\end{aligned}
$$

Given that $\mathbf{E}=\left(\mathbf{E}_{1}^{\top} \mathbf{E}_{2}^{\top}\right)^{\top}$ is a full rank matrix representing a permutation, the transformation

$$
\mathbf{E}^{\top} \mathbf{P}^{\prime} \mathbf{E} \geq 0
$$

leads to the simpler matrix inequality independent of $\mathbf{E}_{2}$,

$$
\mathbf{P}-\mathbf{E}_{1}^{\top} \mathbf{X} \mathbf{E}_{1} \geq 0 .
$$

The previous condition implies the existence of an ellipsoid $\epsilon_{\mathbf{X}}\left(\boldsymbol{e}_{z}\right)$ that encloses $\epsilon_{\mathbf{P}_{z}}\left(\boldsymbol{e}_{z}\right)$. Minimizing the volume of $\epsilon_{\mathbf{X}}\left(\boldsymbol{e}_{z}\right)$, i.e., maximizing the determinant of the matrix $\mathbf{X}$, will make $\epsilon_{\mathbf{X}}\left(\boldsymbol{e}_{z}\right)$ converge to the minimum volume $\epsilon_{\mathbf{P}_{z}}\left(\boldsymbol{e}_{z}\right)$ as long as no additional conditions over $\mathbf{X}$ are applied.

As a result of the above, Eqn. (26) can be replaced by the convex optimization problem:

Find

$$
\left(\mathbf{X}^{+}, \mathbf{P}^{+}, \mathbf{Y}^{+}\right)=\arg \max _{\mathbf{X}>0, \mathbf{P}>0, \mathbf{Y}} \operatorname{det}(\mathbf{X}),
$$

subject to Eqns. (7) and (30), which guarantees that $\epsilon_{\mathbf{X}}^{+}=$ $\epsilon_{\mathbf{P}_{z}}^{+}$. Note that, since $\mu$ is not present in Eqn. (31), the optimal values $\mathbf{X}^{+}=\mathbf{X}^{+}(\alpha), \mathbf{P}^{+}=\mathbf{P}^{+}(\alpha)$ and $\mathbf{Y}^{+}=$ $\mathbf{Y}^{+}(\alpha)$ will only depend on the chosen value of $\alpha$.

Taking into account the previous comments, the optimal quadratically bounded observer represented by $\mathbf{L}^{\star}=\left(\mathbf{P}^{\star}\right)^{-1} \mathbf{Y}^{\star}$ is given by the solution of the optimization problem in Eqn. (25), with $\epsilon_{\mathbf{X}}^{+}=\epsilon_{\mathbf{P}_{z}}^{+}$and $\mathbf{X}^{+}$being the solution of Eqn. (31).

\section{UAV dynamic equations}

In this section, the UAV model that will be used later to derive the disturbance observer is presented. First, the continuous dynamic motion equations for 3D rigid bodies, formulated in momentum form, expressed in the world reference frame and considering the action of gravity, wrench actuators and disturbances, are presented. To the best of the authors' knowledge, this choice, which has the benefit of making the system linear, has not been made before in the field of disturbance estimation in UAVs. Second, a simple but general disturbance model is reviewed. Finally, the joint model taking into account the previous two dynamics is shown.

3.1. Dynamic motion equations. Let the frame, $\{W\}$ be an inertial one with the origin on the earth surface with a given fixed orientation. The NED (north-east-down) configuration is used here as a usual convention, i.e., the $x$-axis of the frame points towards the north pole, the $y$-axis points to the east and the $z$-axis points down, in the direction of the Earth's center.

Let the frame $\{B\}$ be a non-inertial frame centred in the body's centre of mass and rigidly attached to it with the $x$-axis pointing to the front, the $y$-axis pointing to the right and the $z$-axis pointing down. Figure 1 shows these reference frames.

Let the quaternion $\stackrel{\circ}{q}=\left[q_{0}, \boldsymbol{q}_{v}^{\top}\right]^{\top}$ define the attitude between frames, such that

$$
{ }^{w} \boldsymbol{r}=\mathbf{R}(\stackrel{\circ}{q})^{b} \boldsymbol{r},
$$

where ${ }^{w} \boldsymbol{r}$ and ${ }^{b} \boldsymbol{r}$ are different representations of a generic vector $\boldsymbol{r} \in \mathbb{R}^{3}$ expressed in the frames $\{W\}$ and $\{B\}$

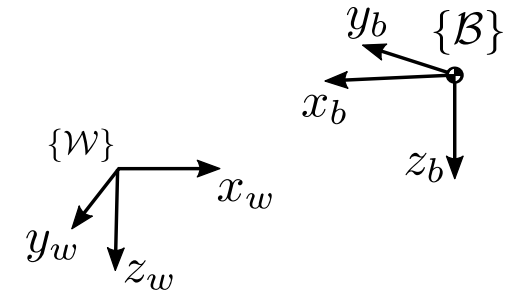

Fig. 1. World and body reference frames depicted. 
respectively, and $\mathbf{R}(\stackrel{\circ}{q}) \in S O(3)$ is a rotation matrix, i.e., $\mathbf{R R}^{\top}=\mathbf{R}^{\top} \mathbf{R}=\mathbf{I}_{3}$, given by

$$
\mathbf{R}(\stackrel{\circ}{q})=\left(q_{0}-\boldsymbol{q}_{v}^{\top} \boldsymbol{q}_{v}\right) \mathbf{I}_{3}+2\left(\boldsymbol{q}_{v} \boldsymbol{q}_{v}^{\top}\right)+2 q_{0}\left[\boldsymbol{q}_{v}\right]_{\times},
$$

where $\left[\boldsymbol{q}_{v}\right]_{\times}$is a skew symmetric matrix for which $\boldsymbol{q}_{v} \times$ $\boldsymbol{r}=\left[\boldsymbol{q}_{v}\right]_{\times} \boldsymbol{r}$.

The equations of motion for a rigid body expressed in the frame $\{W\}$ using the system's linear momentum and spin momentum are

$$
{ }^{w} \dot{\boldsymbol{p}}=\left(\begin{array}{c}
m^{w} \boldsymbol{g}+\mathbf{R}(\stackrel{\circ}{q})^{b} \boldsymbol{f}_{a}+{ }^{w} \boldsymbol{f}_{e} \\
\mathbf{R}(\stackrel{\circ}{q})^{b} \boldsymbol{\tau}_{a}+{ }^{w} \boldsymbol{\tau}_{e}
\end{array}\right),
$$

where ${ }^{w} \boldsymbol{p} \in \mathbb{R}^{6}$ is the lumped momentum, defined as

$$
{ }^{w} \boldsymbol{p}=\left(\begin{array}{c}
m^{w} \boldsymbol{v} \\
{ }^{w} \mathbf{J}^{w} \boldsymbol{\omega}
\end{array}\right)
$$

the vector ${ }^{w} \boldsymbol{v} \in \mathbb{R}^{3}$ defines the relative velocity of the origin of $\{B\}$ with respect to the origin of $\{W\}$, expressed in $\{W\}$ coordinates; ${ }^{w} \boldsymbol{\omega} \in \mathbb{R}^{3}$ represents the relative angular velocity of $\{B\}$ with respect to $\{W\}$, also in $\{W\}$ coordinates; $m \in \mathbb{R}$ denotes the body's mass; ${ }^{w} \mathbf{J} \in \mathbb{R}^{3}$ is the inertia of the body as seen from $\{W\}$, which is related to constant inertia of the body ${ }^{b} \mathbf{J}$ by ${ }^{w} \mathbf{J}(\stackrel{q}{q})=$ $\mathbf{R}(\stackrel{q}{q})^{b} \mathbf{J R}(\stackrel{q}{q})^{\top} ;{ }^{w} \boldsymbol{g} \in \mathbb{R}^{3}$ is the gravity's acceleration vector, known in $\{W\} ;{ }^{b} \boldsymbol{f}_{a} \in \mathbb{R}^{3}$ and ${ }^{b} \boldsymbol{\tau}_{a} \in \mathbb{R}^{3}$ are the actuator force and torque vectors, which are naturally described in the body frame; finally, ${ }^{w} \boldsymbol{f}_{e} \in \mathbb{R}^{3}$ and ${ }^{w} \boldsymbol{\tau}_{e} \in \mathbb{R}^{3}$ are the unknown external forces and torques acting on the platform.

Equation (34) can be written in a compact way as

$$
{ }^{w} \dot{\boldsymbol{p}}=\left(m \boldsymbol{g}^{\prime}+\mathbf{R}^{\prime}(\stackrel{\circ}{q}) \boldsymbol{w}_{a}+\boldsymbol{d}\right),
$$

where

$$
\boldsymbol{g}^{\prime}=\left({ }^{w} \boldsymbol{g}^{\top}, 0,0,0\right)^{\top}
$$

is the extended gravity vector,

$$
\mathbf{R}^{\prime}(\stackrel{\circ}{q})=\left(\begin{array}{cc}
\mathbf{R}(\stackrel{\circ}{q}) & \mathbf{0} \\
\mathbf{0} & \mathbf{R}(\stackrel{\circ}{q})
\end{array}\right)
$$

is an augmented rotation matrix,

$$
\boldsymbol{w}_{a}^{\top}=\left({ }^{b} \boldsymbol{f}_{a}^{\top},{ }^{b} \boldsymbol{\tau}_{a}^{\top}\right)
$$

is the actuator wrench and

$$
\boldsymbol{d}=\Delta \boldsymbol{w}_{a}+\Delta \boldsymbol{g}^{\prime}+\left(\begin{array}{c}
{ }^{w} \boldsymbol{f}_{e} \\
{ }^{w} \boldsymbol{\tau}_{e}
\end{array}\right)
$$

is a vector representing the disturbances acting on the system, which may contain not only the effect of the external wrench but also that of unmodelled dynamics, represented by $\Delta \boldsymbol{w}_{a}$ and $\Delta \boldsymbol{g}^{\prime}$.
3.2. Disturbance model. The dynamics of $\boldsymbol{d}$ are generally unknown and this is the main reason why works like those of Ruggiero et al. (2014) and Yüksel et al. (2014) assume that $\dot{\boldsymbol{d}}=0$. However, this constraint could slow down the estimation output in the case of fast varying dynamics. In order to alleviate this drawback, it could be assumed that the disturbance $\boldsymbol{d}$ is a continuous function driven by an unknown time varying exogenous input $\boldsymbol{\eta}(t)$ at its $r$-th derivative,

$$
\stackrel{(r)}{\boldsymbol{d}}=\boldsymbol{\eta}(t)
$$

as suggested by Kim et al. (2010) and later used by $\mathrm{Su}$ et al. (2015).

In this case, it is interesting to consider an augmented version of the disturbance vector, given by

$$
\boldsymbol{d}_{v}=\left(\boldsymbol{d}^{\top}, \dot{\boldsymbol{d}}^{\top}, \cdots, \boldsymbol{d}^{\top}\right)^{\top}
$$

with the dynamics of $\boldsymbol{d}_{v}$ described by

$$
\dot{\boldsymbol{d}}_{v}=\mathbf{F} \boldsymbol{d}_{v}+\mathbf{G} \boldsymbol{\eta}_{1}
$$

where $\mathbf{F} \in \mathbb{R}^{6(r-1) \times 6(r-1)}$ is given by

$$
\mathbf{F}=\left(\begin{array}{l|l}
\mathbf{0}_{6 r \times 6} & \mathbf{I}_{6(r-1)} \\
\hline \mathbf{0}_{6 \times 6(r-1)}
\end{array}\right)
$$

and $\mathbf{G} \in \mathbb{R}^{6(r-1) \times 6}$ is defined as

$$
\mathbf{G}=\left(\frac{\mathbf{0}_{6(r-1) \times 6}}{\mathbf{I}_{6}}\right) \text {. }
$$

The disturbance vector $\boldsymbol{d}$ can be recovered any time from $\boldsymbol{d}_{v}$ as $\boldsymbol{d}=\mathbf{H} \boldsymbol{d}_{v}$, where $\mathbf{H} \in \mathbb{R}^{6 \times 6(r-1)}$ is another selection matrix defined by

$$
\mathbf{H}=\left(\mathbf{I}_{6}, \mathbf{0}_{6 \times 6(r-1)}\right) .
$$

3.3. Extended model considering disturbances. The augmented dynamic model with state $\boldsymbol{x}^{\top}=\left({ }^{w} \boldsymbol{p}^{\top}, \boldsymbol{d}_{v}^{\top}\right)$ and dynamics given by (36) and (43) can be summarized as

$$
\dot{\boldsymbol{x}}=\left(\begin{array}{c}
{ }^{w} \dot{\boldsymbol{p}} \\
\dot{\boldsymbol{d}}_{v}
\end{array}\right)=\left(\begin{array}{c}
m \boldsymbol{g}^{\prime}+\mathbf{R}^{\prime}(\stackrel{\circ}{q}) \boldsymbol{w}_{a}+\mathbf{H} \boldsymbol{d}_{v} \\
\mathbf{F} \boldsymbol{d}_{v}+\mathbf{G} \boldsymbol{\eta}_{1}
\end{array}\right)
$$

Let $\stackrel{\circ}{q}$ and $\boldsymbol{w}_{a}$ be known and let $\boldsymbol{\eta}_{1}=\boldsymbol{\eta}_{1}(t)$ represent an unknown input; then the dynamics in 47) are linear and can be rewritten as

$$
\dot{\boldsymbol{x}}=\mathbf{A} \boldsymbol{x}+\mathbf{B} \boldsymbol{u}+\mathbf{W}_{1} \boldsymbol{\eta}_{1}
$$

where

$$
\mathbf{A}=\left(\begin{array}{l|l}
\mathbf{0}_{6(r+1) \times 6} & \mathbf{H} \\
\hline \mathbf{F}
\end{array}\right),
$$




$$
\begin{gathered}
\mathbf{B}=\left(\begin{array}{c}
\mathbf{I}_{6} \\
\mathbf{0}_{6 r \times 6}
\end{array}\right), \\
\mathbf{W}_{1}=\left(\begin{array}{c}
\mathbf{0}_{6 \times 6} \\
\mathbf{G}
\end{array}\right),
\end{gathered}
$$

and

$$
\boldsymbol{u}=m \boldsymbol{g}^{\prime}+\mathbf{R}^{\prime}(\stackrel{\circ}{q}) \boldsymbol{w}_{a} .
$$

3.4. System's measurements. In the case of typical navigation applications, an upper-layer estimator is used to reconstruct attitude and velocity states while filtering and removing the bias from the sensors' readings; see, for example, the works of Santamaria-Navarro et al. (2015), Lynen et al. (2013) or Kelly and Sukhatme (2011). As it is a common practice, we will assume in this work that $\stackrel{q}{q},{ }^{w} \boldsymbol{v},{ }^{b} \boldsymbol{\omega}$ and the measurements from accelerometers represented by

$$
{ }^{b} \boldsymbol{a}=\frac{{ }^{b} \boldsymbol{f}_{a}+\mathbf{R}^{\top}(\stackrel{\circ}{q})^{w} \boldsymbol{f}_{\mathrm{ext}}}{m}
$$

are known at regular time intervals. Moreover, the actuator wrench $\boldsymbol{w}_{a}$ is also assumed known.

In this work, these data will be used to construct the input vector $\boldsymbol{u}$ provide measurements of the system as

$$
\begin{aligned}
\boldsymbol{y} & =\left(\begin{array}{c}
{ }^{w} \boldsymbol{p}+\boldsymbol{\eta}_{p} \\
{ }^{w} \boldsymbol{f}_{\mathrm{ext}}+\boldsymbol{\eta}_{f_{a}}
\end{array}\right) \\
& =\left(\begin{array}{c}
m^{b} \boldsymbol{v} \\
\mathbf{R}\left(\stackrel{\stackrel{q}{q})}{\left({ }^{b} \mathbf{J}^{b} \boldsymbol{\omega}\right)}\right. \\
\mathbf{R}(\stackrel{\leftrightarrow}{q})\left(m^{b} \boldsymbol{a}+{ }^{b} \boldsymbol{f}_{a}\right)
\end{array}\right)+\boldsymbol{\eta}_{2} .
\end{aligned}
$$

with $\boldsymbol{\eta}_{2}=\boldsymbol{\eta}_{2}(t)$ representing the measurement errors. From the previous equation, the linear measurement model can be formulated as

$$
\boldsymbol{y}=\mathbf{C} \boldsymbol{x}+\mathbf{W}_{2} \boldsymbol{\eta}_{2},
$$

with

$$
\mathbf{C}=\left(\begin{array}{ll}
\mathbf{I}_{9} & \mathbf{0}_{9 \times 6 r+3}
\end{array}\right)
$$

and

$$
\mathbf{W}_{2}=\mathbf{I}_{9} .
$$

\section{UAV optimal quadratic boundedness observer}

In this section, we use the design procedure presented in Section 3 to generate an observer for the UAV represented by Eqns. (48) and (54). To demonstrate the estimation performance, we use the simulation setup in Fig. 2, where a quadrotor with $m=1.023 \mathrm{~kg}$ and ${ }^{b} \mathbf{J}=$ diag $(9.5,9.5,18.6) 10^{-3} \mathrm{kgm}^{2}$ is controlled to perform a hovering flight during the whole simulation. The inputs

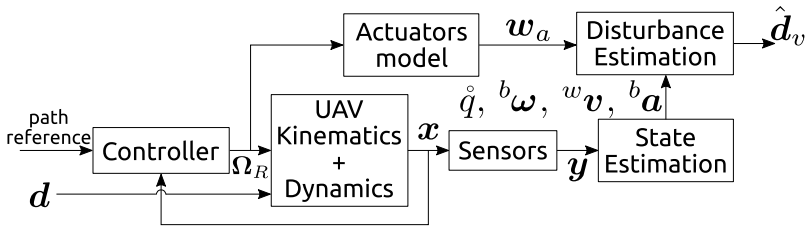

Fig. 2. Simulation setup.

of the dynamic model are the desired angular velocities of the four rotors $\Omega_{R}$ along with the disturbance $\boldsymbol{d}$, whose time plot is given in Fig. 3. The disturbance is a force acting on the world $x$ direction. The set of sensors considered includes an IMU that measures the angular velocity of the UAV and the acceleration of external forces, a tri-axial magnetometer that outputs the Earth's magnetic field direction and a GPS sensor that outputs the position in the $\{W\}$ frame. The controller, the UAV model and the actuator model are a modification of those presented by Hartman et al. (2014), but considering quaternions for attitude parametrization and calibrated to resemble the behaviour of an AscTec Hummingbird quadrotor. The sensor models have been calibrated on the basis of real experiments over the experimental platform.

With this information, a state estimation process produces estimates of the vector $\boldsymbol{y}$ for which the measurement errors have been identified to be inside an ellipsoid $\epsilon_{\mathbf{Q}_{2}}\left(\boldsymbol{\eta}_{2}\right)$ with

$$
\begin{aligned}
& \mathbf{Q}_{2} \\
& =\left(\begin{array}{ccccccccc}
1.5 & -0.3 & 0.3 & 8.3 & -3.2 & -3.1 & -0.2 & -0.02 & -0.1 \\
& 0.7 & -0.1 & -10.5 & 9.3 & -6.2 & 0.04 & -0.03 & -0.01 \\
& & 0.9 & 3.30 & 3.7 & -3.9 & 0.02 & -0.02 & -0.09 \\
& & & 2903 & 111.7 & -277.8 & -2.4 & -0.6 & -0.2 \\
& & & & 4281 & 242.1 & 0.6 & 2.5 & -1.5 \\
& & & & & 951.2 & -1.6 & 1.6 & 1.05 \\
& & & & & & 0.2 & -0.01 & -0.01 \\
& & & & & & & 0.1 & -0.01 \\
& & & & & & & & 0.1
\end{array}\right)
\end{aligned}
$$

Finally, the wrench in the actuators is supposed to be completely known, given that the actuator model reproduces exactly the implementation in the dynamic block.

Given the disturbance in Fig. 3, the value of the order of the disturbance model has been set to $r=$ 3 , while $\mathbf{Q}_{1}=10^{6} \mathbf{I}_{6}$ has been selected big enough to make $\epsilon_{\mathbf{Q}_{1}}\left(\boldsymbol{\eta}_{1}\right)$ sufficiently small while taking into account possible numerical errors on the simulation. As a consequence, the matrix

$$
\mathbf{Q}=\frac{1}{2}\left(\begin{array}{cc}
\mathbf{Q}_{1} & \mathbf{0} \\
\mathbf{0} & \mathbf{Q}_{2}
\end{array}\right)
$$

defines the model and measurement errors ellipsoid in Eqn. (54). 


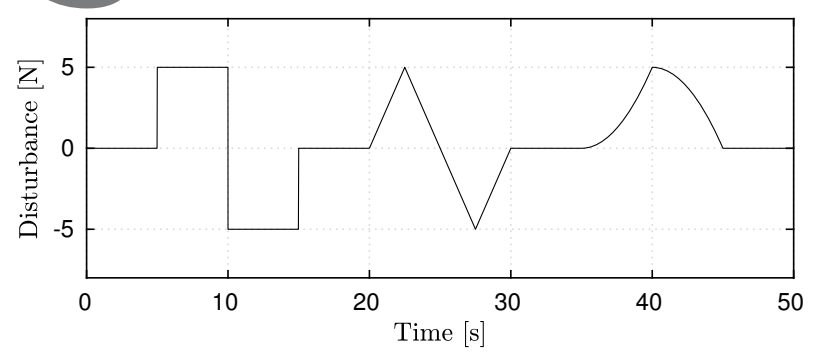

Fig. 3. Disturbance applied to the quadrotor. The first part is a succession of constant signals (from $t=5 \mathrm{~s}$ to $t=15 \mathrm{~s}$ ). The second part is a triangular signal (from $t=20 \mathrm{~s}$ to $t=30 \mathrm{~s}$ ) and the third one is a quadratic signal (from $t=35 \mathrm{~s}$ to $t=45 \mathrm{~s}$ ).

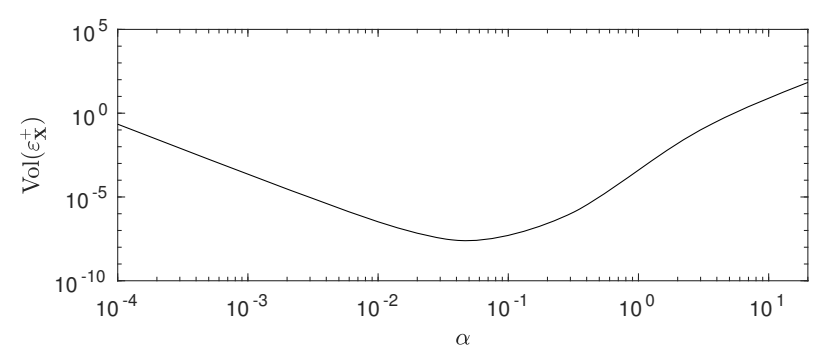

Fig. 4. Minimum value of $\operatorname{Vol}\left(\epsilon_{\mathbf{X}}^{+}\right)$as a function of $\alpha$.

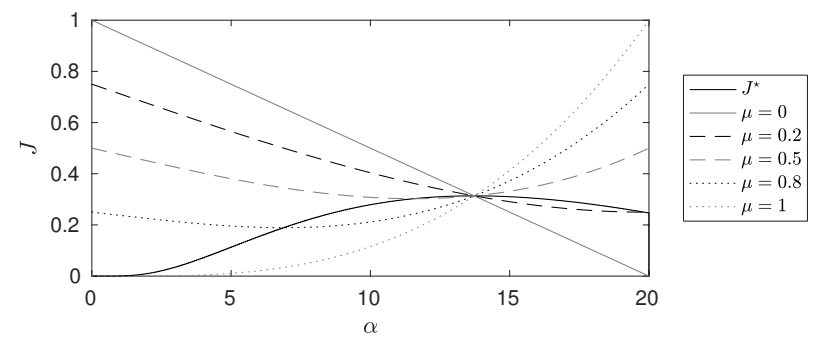

Fig. 5. Values of the objective function as a function of $\alpha$ for fixed $\mu$ and an optimal value of the objective function.

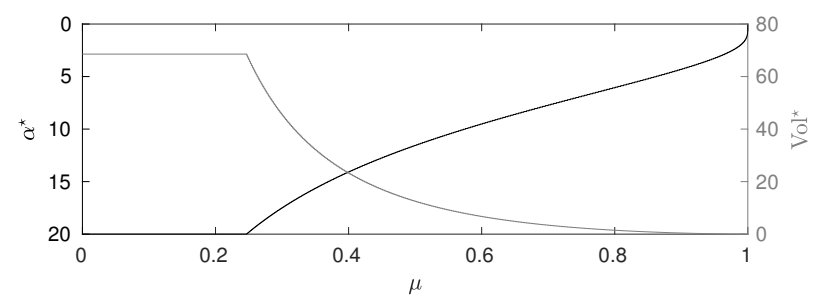

Fig. 6. Optimal values of the convergence rate and ultimate bounding volume as a function of the design parameter.

Since in this case the ultimate goal is to estimate only the disturbances $\boldsymbol{d}$ whereas the exact values of the remaining states are irrelevant, $\mathbf{E}_{1}$ has been chosen as

$$
\mathbf{E}_{1}=\left(\mathbf{0}_{6 \times 6}, \mathbf{H}\right) .
$$

By using the previous data, the optimization problem in Eqn. (31) has been solved for values of $\alpha \in[0,20]$.
The optimal volume of the produced ellipsoids $\epsilon_{\mathbf{X}}^{+}(\alpha)$ is shown in Fig. 4

The results of the objective function for several values of $\mu$ along with the optimal value as a function of $\alpha$ can be found in Fig. 5

Finally, the optimal values of the unnormalized objectives $\alpha^{\star}$ and $\operatorname{Vol}\left(\epsilon_{\mathbf{X}}^{+}\right)$as a function of the design parameter $\mu$ are shown in Fig. 6

Disturbance estimation performance for the values of the decision variable $\mu=0.2$ and $\mu=0.95$ are shown in Fig. 7. Note that the estimation of the disturbance is not straightforward since in the abrupt changes in the signal the derivatives are unbounded by definition. However, even in this case, the filter design guarantees the error stability and, by comparing the two outputs, it can be appreciated that, when the hypothesis of the filter (in this case $\dddot{d} \in \epsilon_{\mathbf{Q}_{1}}\left(\boldsymbol{\eta}_{1}\right)$ ) are fulfilled, the estimation corresponding to higher $\mu$ is slower than the one with a lower value of $\mu$, while it converges to a smaller region in the neighbourhood of the true signal, as can also be observed in Fig. 6

\section{Conclusions}

The design procedure presented in the first part of the paper is a generic development devoted to generate state observers for linear uncertain systems. The presented results allow deriving observers that guarantee an optimal trade-off between minimum volume error solutions and a maximum convergence rate. Considering the volume of the solution as an objective to minimize is an idea that has not been presented in the literature before. In the modelling part, we have presented a linear model for a quadrotor which considers a possible high order disturbance acting over the aircraft as part of the state.

It should be assumed that such a linear model for a quadrotor is not a simplification but rather an exact result obtained after considering a proper state and reference frames. Both the model and theory meet to formulate a state observer which ensures the stability of the estimation while minimizing the error associated to the disturbance. The example considered exhibits the effect of the decision variable in the design, and the results demonstrate the performance of the observer for different choices of the design parameter estimating a disturbance.

The quadrotor model and the extended system used to estimate disturbances in the second part of the article can be employed along with other state estimation designs based on ISS or $H_{\infty}$, among others, to provide different estimation observers. In a future work we plan to make an analysis of these methods to show explicitly how they affect the minimum volume of the error ellipsoid as well as the decaying rate and provide a fair comparison with the results presented here. Future works will also include experimental results using a real UAV platform. 

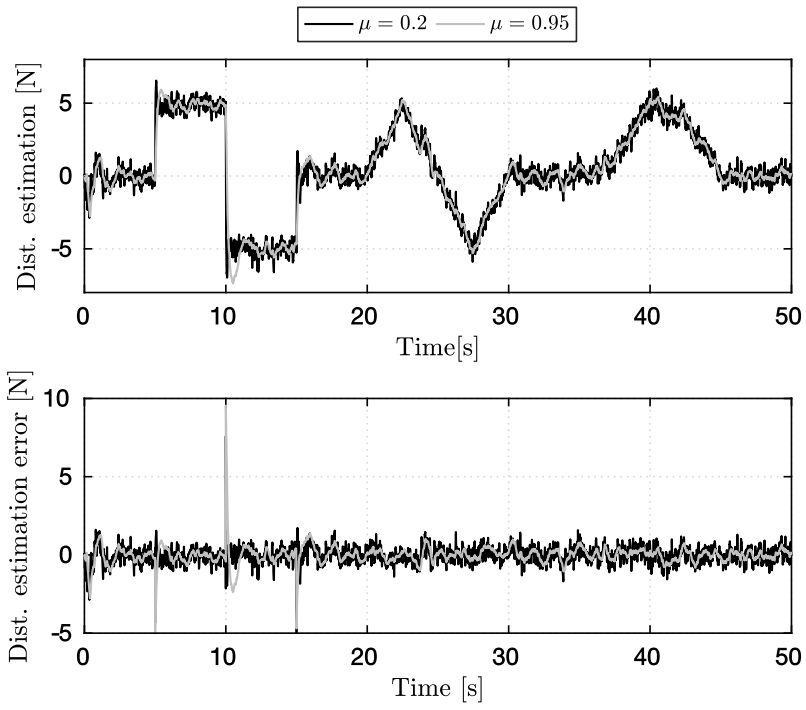

Fig. 7. Disturbance estimation and errors.

\section{Acknowledgment}

This work has been partially funded by the Spanish Government and FEDER through the projects CICYT DEOCS and SCAV (refs. MINECO DPI2016-76493, DPI2017-88403-R). It has also been partially funded by AGAUR of Generalitat de Catalunya through the Advanced Control Systems (SAC) group grant (2017 SGR 482), and by AGAUR and the Spanish Research Agency through the Maria de Maetzu Seal of Excellence to IRI (MDM-2016-0656). Julian Cayero is also supported by Secretaria d'Universitats i Recerca del Departament d'Empresa i Coneixement de la Generalitat de Catalunya, the European Social Fund (ESF) and the Agency for Management of University and Research Grants (AGAUR) under an FI-DGR grant (ref. 2016FI_B1 00024). Damiano Rotondo is also supported by a Juan de la Cierva-Formacion grant (FJCI-2016-29019).

\section{References}

Abbaszadeh, M. and Marquez, H.J. (2009). LMI optimization approach to robust $H_{\infty}$ observer design and static output feedback stabilization for discrete-time nonlinear uncertain systems, International Journal of Robust and Nonlinear Control 19(3): 313-340.

Alessandri, A. (2004). Observer design for nonlinear systems by using input-to-state stability, 43rd IEEE Conference on Decision and Control, 2004. CDC, Nassau, The Bahamas, Vol. 4, pp. 3892-3897.

Alessandri, A., Baglietto, M. and Battistelli, G. (2004). On estimation error bounds for receding-horizon filters using quadratic boundedness, IEEE Transactions on Automatic Control 49(8): 1350-1355.

Alessandri, A., Baglietto, M. and Battistelli, G. (2006). Design of state estimators for uncertain linear systems using quadratic boundedness, Automatica 42(3): 497-502.
Alessandri, A. (2013). Design of time-varying state observers for nonlinear systems by using input-to-state stability, American Control Conference (ACC), Washington, DC, USA, pp. 280-285.

Brockman, M.L. and Corless, M. (1995). Quadratic boundedness of nonlinear dynamical systems, 34th IEEE Conference on Decision and Control, New Orleans, LA, USA, Vol. 1, pp. 504-509.

Brockman, M.L. and Corless, M. (1998). Quadratic boundedness of nominally linear systems, International Journal of Control 71(6): 1105-1117.

Buciakowski, M., Witczak, M., Mrugalski, M. and Theilliol, D. (2017a). A quadratic boundedness approach to robust DC motor fault estimation, Control Engineering Practice 66: $181-194$

Buciakowski, M., Witczak, M., Puig, V., Rotondo, D., Nejjari, F. and Korbicz, J. (2017b). A bounded-error approach to simultaneous state and actuator fault estimation for a class of nonlinear systems, Journal of Process Control 52: $14-25$.

Busawon, K.K. and Kabore, P. (2001). Disturbance attenuation using proportional integral observers, International Journal of Control 74(6): 618-627.

Chadli, M. and Karimi, H.R. (2013). Robust observer design for unknown inputs Takagi-Sugeno models, IEEE Transactions on Fuzzy Systems 21(1): 158-164.

Chen, W.-H., Ballance, D.J., Gawthrop, P.J. and O'Reilly, J. (2000). A nonlinear disturbance observer for robotic manipulators, IEEE Transactions on Industrial Electronics 47(4): 932-938.

Chen, W.-H., Yang, J., Guo, L. and Li, S. (2016). Disturbance-observer-based control and related methods-an overview, IEEE Transactions on Industrial Electronics 63(2): 1083-1095.

Chibani, A., Chadli, M., Shi, P. and Braiek, N.B. (2017). Fuzzy fault detection filter design for T-S fuzzy systems in the finite-frequency domain, IEEE Transactions on Fuzzy Systems 25(5): 1051-1061.

Ding, B. (2009). Quadratic boundedness via dynamic output feedback for constrained nonlinear systems in Takagi-Sugeno's form, Automatica 45(9): 2093-2098.

Ding, B. (2013). New formulation of dynamic output feedback robust model predictive control with guaranteed quadratic boundedness, Asian Journal of Control 15(1): 302-309.

Ding, B. and Pan, H. (2016). Output feedback robust MPC for LPV system with polytopic model parametric uncertainty and bounded disturbance, International Journal of Control 89(8): 1554-1571.

Godbole, A.A., Kolhe, J.P. and Talole, S.E. (2013). Performance analysis of generalized extended state observer in tackling sinusoidal disturbances, IEEE Transactions on Control Systems Technology 21(6): 2212-2223.

Grip, H.F., Saberi, A. and Johansen, T.A. (2012). Observers for interconnected nonlinear and linear systems, Automatica 48(7): 1339-1346. 
Hartman, D., Landis, K., Mehrer, M., Moreno, S. and Kim, J. (2014). Quadcopter dynamic modeling and simulation (Quad-Sim), https://github.com/dch33/QuadSim.

Hassani, H., Zarei, J., Chadli, M. and Qiu, J. (2017). Unknown input observer design for interval type-2 T-S fuzzy systems with immeasurable premise variables, IEEE Transactions on Cybernetics 47(9): 2639-2650.

Johnson, C. (1971). Accommodation of external disturbances in linear regulator and servomechanism problems, IEEE Transactions on Automatic Control 16(6): 635-644.

Kelly, J. and Sukhatme, G. S. (2011). Visual-inertial sensor fusion: Localization, mapping and sensor-to-sensor self-calibration, The International Journal of Robotics Research 30(1): 56-79.

Kim, K.-S., Rew, K.-H. and Kim, S. (2010). Disturbance observer for estimating higher order disturbances in time series expansion, IEEE Transactions on Automatic Control 55(8): 1905-1911.

Koenig, D. (2005). Unknown input proportional multiple-integral observer design for linear descriptor systems: Application to state and fault estimation, IEEE Transactions on Automatic Control 50(2): 212-217.

Kwon, S. and Chung, W. K. (2003). A discrete-time design and analysis of perturbation observer for motion control applications, IEEE Transactions on Control Systems Technology 11(3): 399-407.

Li, S., Yang, J., Chen, W.-H. and Chen, X. (2012). Generalized extended state observer based control for systems with mismatched uncertainties, IEEE Transactions on Industrial Electronics 59(12): 4792-4802.

Lynen, S., Achtelik, M. W., Weiss, S., Chli, M. and Siegwart, R. (2013). A robust and modular multi-sensor fusion approach applied to MAV navigation, IEEE/RSJ International Conference on Intelligent Robots and Systems (IROS), Tokyo, Japan, pp. 3923-3929.

Ping, X. (2017). Output feedback robust MPC based on off-line observer for LPV systems via quadratic boundedness, Asian Journal of Control 19(4): 1641-1653.

Ping, X., Wang, P. and Li, Z. (2017). Quadratic boundedness of LPV systems via saturated dynamic output feedback controller, Optimal Control Applications and Methods 38(6): 1239-1248.

Miettinen, K. (1999). Nonlinear Multiobjective Optimization, International Series in Operations Research and Management Science, Vol. 12, Springer, New York, NY.

Ros, L., Sabater, A. and Thomas, F. (2002). An ellipsoidal calculus based on propagation and fusion, IEEE Transactions on Systems, Man, and Cybernetics B: Cybernetics 32(4): 430-442.

Rotondo, D., Witczak, M., Puig, V., Nejjari, F. and Pazera, M. (2016). Robust unknown input observer for state and fault estimation in discrete-time Takagi-Sugeno systems, International Journal of Systems Science 47(14): 3409-3424.
Ruggiero, F., Cacace, J., Sadeghian, H. and Lippiello, V. (2014). Impedance control of VToL UAVs with a momentum-based external generalized forces estimator, IEEE International Conference on Robotics and Automation (ICRA), Hong Kong, China, pp. 2093-2099.

Ruggiero, F., Cacace, J., Sadeghian, H. and Lippiello, V. (2015). Passivity-based control of VToL UAVs with a momentum-based estimator of external wrench and unmodeled dynamics, Robotics and Autonomous Systems 72: 139-151.

Santamaria-Navarro, A., Sola, J. and Andrade-Cetto, J. (2015). High-frequency MAV state estimation using low-cost inertial and optical flow measurement units, IEEE/RSJ International Conference on Intelligent Robots and Systems (IROS), Hamburg, Germany, pp. 1864-1871.

She, J.-H., Fang, M., Ohyama, Y., Hashimoto, H. and Wu, M. (2008). Improving disturbance-rejection performance based on an equivalent-input-disturbance approach, IEEE Transactions on Industrial Electronics 55(1): 380-389.

$\mathrm{Su}$, J., Chen, W.-H. and Li, B. (2015). High order disturbance observer design for linear and nonlinear systems, IEEE International Conference on Information and Automation, Lijiang, China, pp. 1893-1898.

Wang, Z., Huang, B. and Unbehauen, H. (1999). Robust $h_{\infty}$ observer design of linear state delayed systems with parametric uncertainty: The discrete-time case, Automatica 35(6): 1161-1167.

Witczak, M., Buciakowski, M. and Aubrun, C. (2016). Predictive actuator fault-tolerant control under ellipsoidal bounding, International Journal of Adaptive Control and Signal Processing 30(2): 375-392.

Witczak, M., Rotondo, D., Puig, V., Nejjari, F. and Pazera, M. (2017). Fault estimation of wind turbines using combined adaptive and parameter estimation schemes, International Journal of Adaptive Control and Signal Processing 32(4): 549-567.

Yüksel, B., Secchi, C., Bülthoff, H.H. and Franchi, A. (2014). A nonlinear force observer for quadrotors and application to physical interactive tasks, IEEE/ASME International Conference on Advanced Intelligent Mechatronics (AIM), Besacon, France, pp. 433-440.

Zeitz, M. (1987). The extended Luenberger observer for nonlinear systems, Systems \& Control Letters 9(2): $149-156$.

Zhang, L., Liu, X. and Kong, X. (2012a). State estimators for uncertain linear systems with different disturbance/noise using quadratic boundedness, Journal of Applied Mathematics 2012, Article ID: 101353.

Zhang, W., Su, H., Wang, H. and Han, Z. (2012b). Full-order and reduced-order observers for one-sided Lipschitz nonlinear systems using Riccati equations, Communications in Nonlinear Science and Numerical Simulation 17(12): 4968-4977.

Zou, T. and Li, S. (2011). Stabilization via extended nonquadratic boundedness for constrained nonlinear 
systems in Takagi-Sugeno's form, Journal of the Franklin Institute 348(10): 2849-2862.

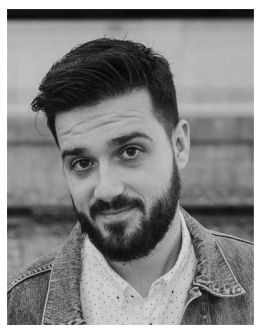

Julian Cayero received the BS and SM degrees in aerospace engineering from Universitat Politècnica de Catalunya (UPC) in 2012. From 2012 to 2015 , he was a research assistant at the Acoustic and Mechanical Engineering Laboratory (LEAM) and the Research Center for Supervision, Safety and Automatic Control (CS2AC), both at UPC. Since March 2015 he has been working toward the $\mathrm{PhD}$ degree with the $\mathrm{Re}$ search Center for Supervision, Safety and Automatic Control (CS2AC) at Universitat Politècnica de Catalunya. His research topics are related to flying vehicles, aerodynamics, control and state estimation.

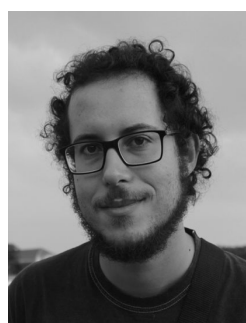

Damiano Rotondo was born in Italy in $1987 . \mathrm{He}$ received the BS degree (with honors) from the Second University of Naples, Italy, the MS degree (with honors) from the University of Pisa, Italy, and the $\mathrm{PhD}$ degree (with honors) from Universitat Politècnica de Catalunya, Spain, in 2008, 2011 and 2016, respectively. Since February 2018, he has been a Juan de la Cierva fellow at the Institute of Robotics and Industrial Informatics (IRI) at the Spanish National Research Council (CSIC). His main research interests include gain-scheduled control systems, fault detection and isolation (FDI) and fault tolerant control (FTC) of dynamic systems. He has published several papers in international conference proceedings and scientific journals.

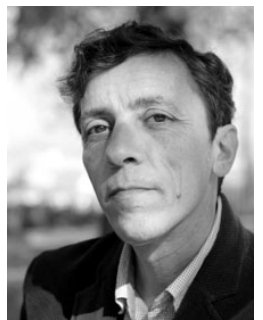

Bernardo Morcego is an associate professor at Universitat Politècnica de Catalunya (UPC). $\mathrm{He}$ received a $\mathrm{PhD}$ degree in computer science from UPC in 2000. He has been teaching several subjects in the area of automatic control in the Schools of Engineering and Aeronautics in Terrassa and Barcelona. He is a member of the Research Center for Supervision, Safety and Automatic Control of UPC. His research interests include UAV control systems, computer vision applications and intelligent control.

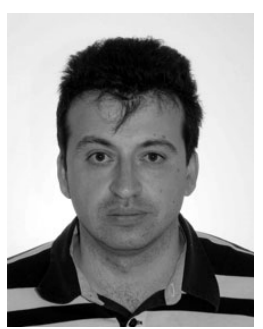

Vicenc Puig was born Spain in 1969. He received the $\mathrm{PhD}$ degree in control engineering in 1999 and the telecommunications engineering degree in 1993, both from Universitat Politècnica de Catalunya (UPC), Barcelona, Spain. He is currently a full professor of automatic control and the leader of the Advanced Control Systems (SAC) research group of the Research Center for Supervision, Safety and Automatic Control (CS2AC) at UPC. His main research interests are in fault detection, isolation and fault-tolerant control of dynamic systems. He has been involved in several European projects and networks, and has published many papers in international conference proceedings and scientific journals.

\section{Appendix}

Given the ellipsoidal set defined by

$$
\epsilon_{\mathbf{P}}(\boldsymbol{x}):=\boldsymbol{x}^{\top} \mathbf{P} \boldsymbol{x} \leq 1,
$$

with $\boldsymbol{x} \in \mathbb{R}^{n}$ and $\mathbf{P}>0$, this section is devoted to finding the projection of this ellipsoid onto the subspace to which the vector $\boldsymbol{z}_{1}$ belongs, $\boldsymbol{z}_{1} \in \mathbb{R}^{m}, m<n$, being a part of the vector $\boldsymbol{x}$, i.e., $\boldsymbol{z}=\mathbf{E}_{1} \boldsymbol{x}$, with $\mathbf{E}_{1} \in$ $\mathbb{R}^{m \times n}$ representing a distribution matrix. This problem is equivalent to finding the ellipsoidal projection of $\epsilon_{\mathbf{P}}(\boldsymbol{x})$ onto the subspace spanned by the rows of $\mathbf{E}_{1}$, denoted by $\left\langle\mathbf{E}_{1}\right\rangle$.

Consider also $\mathbf{E}_{2} \in \mathbb{R}^{l}$ with $l=m-n$ to be an orthogonal complement of $\mathbf{E}_{1}$, i.e., $\left\langle\mathbf{E}_{2}\right\rangle$ is orthogonal to $\left\langle\mathbf{E}_{1}\right\rangle^{\perp}$ and $\boldsymbol{z}_{2}=\mathbf{E}_{2} \boldsymbol{x}$. Then we have

$$
\boldsymbol{z}=\mathbf{E} \boldsymbol{x},
$$

with

$$
\mathbf{E}^{\top}=\left[\begin{array}{ll}
\mathbf{E}_{1}^{\top} & \mathbf{E}_{2}^{\top}
\end{array}\right]
$$

and $\boldsymbol{z}=\left(\boldsymbol{z}_{1}^{\top}, \boldsymbol{z}_{2}^{\top}\right)^{\top}$

Moreover, since matrix $\mathbf{E}$ represents an orthonormal basis of $\mathbb{R}^{n}$, its inverse $\mathbf{E}^{-1}=\mathbf{E}^{\top}$ and, as a consequence,

$$
\boldsymbol{x}=\mathbf{E}^{\top} \boldsymbol{z}=\left[\begin{array}{ll}
\mathbf{E}_{1}^{\top} & \mathbf{E}_{2}^{\top}
\end{array}\right] \boldsymbol{z}=\mathbf{E}_{1}^{\top} \boldsymbol{z}_{1}+\mathbf{E}_{2}^{\top} \boldsymbol{z}_{2} .
$$

The projection of the boundary of the ellipsoid in Eqn. (A1) represented by $\partial \epsilon_{\mathbf{P}}(\boldsymbol{x})$ onto $\left\langle\mathbf{E}_{1}\right\rangle$ describes the boundary of the target ellipsoid $\epsilon_{\mathbf{P}_{z}}\left(\boldsymbol{z}_{1}\right)$ represented as $\partial \epsilon_{\mathbf{P}_{z}}\left(\boldsymbol{z}_{1}\right)$. Consider the scalar function $S=\boldsymbol{x}^{\top} \mathbf{P} \boldsymbol{x}-1$. Then

$$
\partial \epsilon_{\mathbf{P}_{\mathbf{z}}}\left(\boldsymbol{z}_{1}\right):=\left(\boldsymbol{x} \mid S=0, \nabla_{\boldsymbol{x}}(S) \in\left\langle\mathbf{E}_{1}\right\rangle\right)
$$

or, equivalently,

$$
\partial \epsilon_{\mathbf{P}_{\mathbf{z}}}\left(\boldsymbol{z}_{1}\right):=\left(\boldsymbol{x} \mid S=0, \mathbf{E}_{2} \nabla_{\boldsymbol{x}}(S)=\mathbf{0}\right) .
$$

The second condition on the right-hand side of Eqn. (A5) implies that

$$
\mathbf{E}_{2} \mathbf{P} \boldsymbol{x}=0
$$

By substituting Eqn. A4 into the previous relation, a map between $z_{1}$ and $z_{2}$ can be found for points on the boundary as

$$
\boldsymbol{z}_{2}=-\left(\mathbf{E}_{2} \mathbf{P} \mathbf{E}_{2}^{\top}\right)^{-1} \mathbf{E}_{2} \mathbf{P} \mathbf{E}_{1}^{\top} \boldsymbol{z}_{1} .
$$

The previous result, along with Eqn. (A4), can be substituted in $\boldsymbol{x}^{\top} \mathbf{P} \boldsymbol{x}=1$, leading to

$$
\partial \epsilon_{\mathbf{P}_{\mathbf{z}}}\left(\boldsymbol{z}_{1}\right):=\left(\boldsymbol{z}_{1} \mid \boldsymbol{z}_{1}^{\top} \mathbf{P}_{z} \boldsymbol{z}_{1}=1\right),
$$

with $\mathbf{P}_{z}=\mathbf{E}_{1} \mathbf{P} \mathbf{E}_{1}^{\top}-\mathbf{E}_{1} \mathbf{P} \mathbf{E}_{2}^{\top}\left(\mathbf{E}_{2} \mathbf{P} \mathbf{E}_{2}^{\top}\right)^{-1} \mathbf{E}_{2} \mathbf{P} \mathbf{E}_{1}^{\top}$.

Since $\epsilon_{\mathbf{P}_{\mathbf{z}}}\left(\boldsymbol{z}_{1}\right)$ must contain the origin as $\epsilon_{\mathbf{P}}(\boldsymbol{x})$ did, $\epsilon_{\mathbf{P}_{\mathbf{z}}}\left(\boldsymbol{z}_{1}\right)$ will be defined by

$$
\epsilon_{\mathbf{P}_{\mathbf{z}}}\left(\boldsymbol{z}_{1}\right):=\left(\boldsymbol{z}_{1} \mid \boldsymbol{z}_{1}^{\top} \mathbf{P}_{z} \boldsymbol{z}_{1} \leq 1\right) .
$$

Received: 15 December 2017 Revised: 21 May 2018 Accepted: 16 December 2018 